\title{
Universiteit
}

Leiden

The Netherlands

\section{Complete elastic tensor through the first-order transformation in} U2Rh3Si5

Leisure, R.G.; Kern, S.; Drymiotis, F.R.; Ledbetter, H.; Migliori, A.; Mydosh, J.A.

\section{Citation}

Leisure, R. G., Kern, S., Drymiotis, F. R., Ledbetter, H., Migliori, A., \& Mydosh, J. A. (2005). Complete elastic tensor through the first-order transformation in U2Rh3Si5. Physical Review Letters, 95(7), 075506. doi:10.1103/PhysRevLett.95.075506

Version: $\quad$ Not Applicable (or Unknown)

License: $\quad$ Leiden University Non-exclusive license

Downloaded from: https://hdl.handle.net/1887/62836

Note: To cite this publication please use the final published version (if applicable). 


\title{
Complete Elastic Tensor through the First-Order Transformation in $\mathbf{U}_{2} \mathbf{R h}_{3} \mathbf{S i}_{5}$
}

\author{
R. G. Leisure, ${ }^{1}$ S. Kern, ${ }^{1}$ F. R. Drymiotis, ${ }^{2}$ H. Ledbetter,${ }^{2}$ A. Migliori, ${ }^{2}$ and J. A. Mydosh ${ }^{3}$ \\ ${ }^{1}$ Department of Physics, Colorado State University, Fort Collins, Colorado 80523, USA \\ ${ }^{2}$ Los Alamos National Laboratory, Los Alamos, New Mexico 87545, USA \\ ${ }^{3}$ Kamerlingh Onnes Laboratory, Leiden University, 2300RA Leiden, \\ The Netherlands/Max Planck Institute for Chemical Physics of Solids, 01187 Dresden, Germany
}

(Received 8 March 2005; published 12 August 2005)

\begin{abstract}
The complete elastic tensor of $\mathrm{U}_{2} \mathrm{Rh}_{3} \mathrm{Si}_{5}$ has been determined over the temperature range of 5-300 $\mathrm{K}$, including the dramatic first-order transition to an antiferromagnetic state at $25.5 \mathrm{~K}$. Sharp upward steps in the elastic moduli as the temperature is decreased through the transition reveal the first-order nature of the phase change. In the antiferromagnetic state the temperature dependence of the elastic moduli scales with the square of the ordered moment on the uranium ion, demonstrating strong spin-lattice coupling. The temperature dependence of the moduli well above the transition indicates coupling of the ultrasonic waves to the crystal electric field levels of the uranium ion where the lowest state is a singlet. The elastic constant data suggest that the first-order phase change is magnetically driven by a bootstrap mechanism involving the ground state singlet and a magnetically active crystal electric field level.
\end{abstract}

PACS numbers: 62.20.Dc, 64.70.-p, 75.50.Ee

Strongly correlated or heavy-electron uranium intermetallic compounds remain a topic of considerable interest with a variety of puzzles waiting to be completed $[1,2]$. Because of the multiple ( 2 or 3 ) $5 f$ electrons of uranium and their strong hybridization, a "duality" results [3] with partially localized and partial itinerant $5 f$ electrons that further interact with the ligand $s, p, d$ electrons of the compound. Here it is most difficult to treat this situation theoretically and even more so experimentally where such basic properties as the $\mathrm{U}$ valency and the corresponding crystal-field levels are arduous or impossible to ascertain. However, there exist a small number of $U$ intermetallics where the correlation/hybridization effects are much weaker so that mostly localized atomic ground states with crystalline electric field (CEF) levels can be observed. By studying these contrasting systems one can learn more about the $\mathrm{U}$ correlations and the conditions for hybridization in the multi $5 f$ electron actinides.

The prime example here is $\mathrm{UPd}_{3}$ in which multiple combinations of magnetic (spin) dipole and quadrupole ordering transitions have been found [4,5]. In particular, recent synchrotron diffraction experiments have distinguished the close, but still resolvable, orbital and spin orderings [6]. $\mathrm{UPd}_{3}$ represents a rare realization of welllocalized $5 f$ electrons and long-range quadrupole interactions. Another enigmatic, well-localized U compound is $\mathrm{U}_{2} \mathrm{Rh}_{3} \mathrm{Si}_{5}$. Discovered [7] in 1990, this quasiorthorhombic compound exhibits a single, dramatic first-order phase transition at $25.5 \mathrm{~K}$ in all bulk properties [8,9]. $\left(\mathrm{U}_{2} \mathrm{Rh}_{3} \mathrm{Si}_{5}\right.$ forms in the monoclinic structure, but the monoclinic distortion is so small that the compound is commonly represented in quasiorthorhombic symmetry.) Neutron diffraction has determined a canted antiferromagnetic structure of tilted double axis Ising-like spins with projected moments of 1.3 ( $a$ axis) and 1.9 ( $b$ axis) $\mu_{B}$ per $\mathrm{U}$ atom [10] (see Fig. 1). A sharp steplike metamagnetic transition occurs at $14 \mathrm{~T}$ causing the $\mathrm{U}$ spin to fully align along the $b$ axis with a saturation magnetization of $1.8 \mu_{B}$ per $U$ atom. Attempts at resolving the CEF scheme are ambiguous and require further effort [11]. Although various models have been speculated [12] for the unusual first-order transition, its true nature remains unknown. Yet, in all cases, a strong spin-lattice coupling seems necessary.

In this Letter we present a complete resonant ultrasound spectroscopy [13] study of the nine independent elastic constants associated with the quasiorthorhombic structure of $\mathrm{U}_{2} \mathrm{Rh}_{3} \mathrm{Si}_{5}$. We have determined all nine elastic constants and their temperature dependences both above and, surprisingly, below the transition. Full elastic-coefficient tensors for both phases across a phase transformation have been reported for only a handful of materials $[14,15]$. Our ultrasonic results not only confirm the first-order nature of the phase transition, but also clearly demonstrate the strong spin-lattice coupling by the exact moduli scaling to the square of the sublattice magnetization. In addition, we

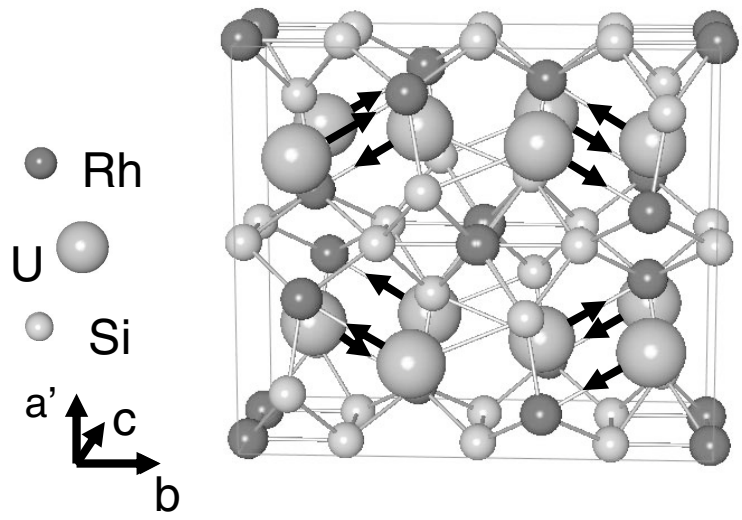

FIG. 1. Crystal structure of $\mathrm{U}_{2} \mathrm{Rh}_{3} \mathrm{Si}_{5}$ in the quasiorthorhombic lattice. The arrows indicate the magnetic moments in the ordered state. 
have derived the CEF level scheme and thereby clarified the driving mechanism for the massive phase transition.

The experimental results reported here were made on a single-crystal specimen with room-temperature dimensions of $2.389,1.755$, and $2.189 \mathrm{~mm}$ along the quasiorthorhombic axes $a^{\prime}, b$, and $c$, respectively. After initial measurements on a larger specimen, one dimension was substantially reduced as a check on the assignments of the elastic constants. The lowest 46 resonances were fit to determine the elastic constants with a maximum rms error of $0.24 \%$, this value being almost independent of temperature.

Figure 2 shows all nine independent elastic moduli $C_{i j}$ over the temperature range of 5-300 K. Here we discuss only a few features from the data of Fig. 2; a much more detailed analysis will be given later. From the lowtemperature elastic constants a Debye temperature of $\Theta_{D}=378 \mathrm{~K}$ is calculated. For a silicide, this number is moderately high, reflecting moderately rigid interatomic bonding. From a Blackman diagram [16,17] (a plot of reduced $C_{i j}$ elastic constants which reveals trends in interatomic bonding), it is found that the compound lies near the line for elastic isotropy: $A=2 C_{44} /\left(C_{11}-C_{12}\right)=1$, indicating overall near-isotropic interatomic interactions. The

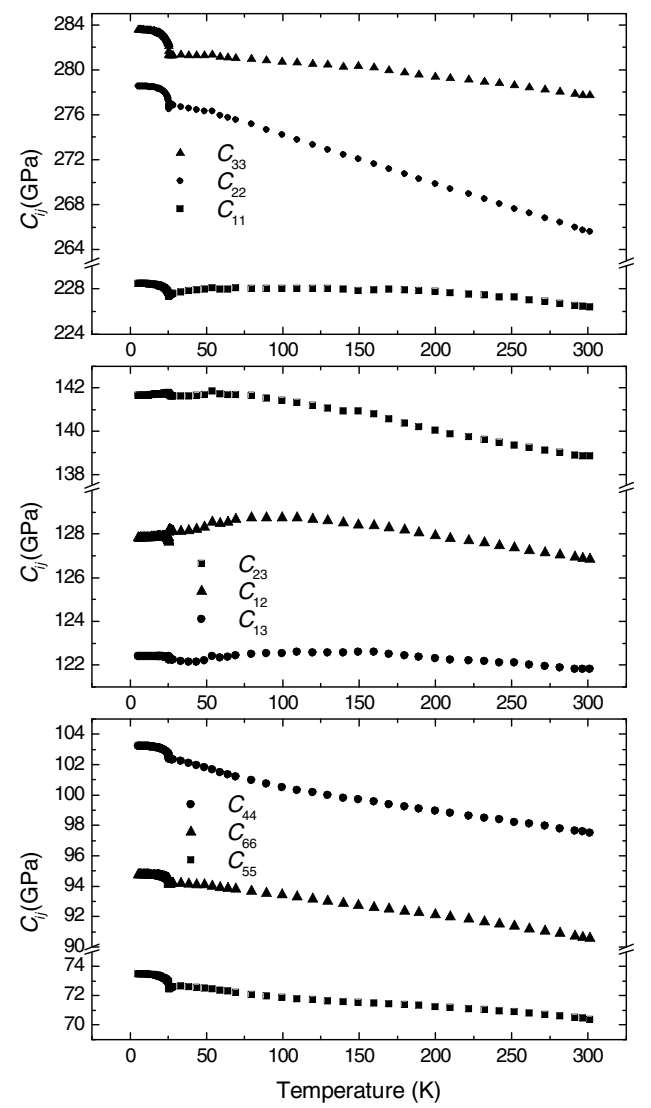

FIG. 2. The nine independent elastic constants of quasiorthorhombic $\mathrm{U}_{2} \mathrm{Rh}_{3} \mathrm{Si}_{5}$ vs temperature as determined by resonant ultrasound spectroscopy. All nine $C_{i j}$ show abnormal behavior. The dashed line in Fig. 3(c) illustrates normal behavior. At $25.5 \mathrm{~K}$, transformation occurs to the antiferromagnetic state. position of the compound in the plot indicates bonding intermediate between strong ionic and strong covalent, slightly favoring covalency.

We now focus on the phase transition and choose one elastic constant, $C_{55}$, to illustrate the salient points. Figure 3 gives the data for this modulus at various temperature scales. There are three points we wish to discuss. First, the $C_{55}$ modulus shows an abrupt step upward at the phase transition. This behavior is characteristic of all six diagonal moduli. The Landau theory of second-order phase transitions predicts an elastic constant decrease on entering the ordered phase for coupling between order parameter and strain that is quadratic in the order parameter $[18,19]$. The coupling is expected to be quadratic in the order parameter in the present case (additional evidence in presented in the following paragraph), because it should not matter if the sublattice magnetization is shifted by half a wavelength (equivalent to a sign reversal). Thus, the upward step of Fig. 3 provides strong evidence for the first-order character of the transition.

The second point involves a comparison of the modulus measurements with neutron scattering measurements [10].

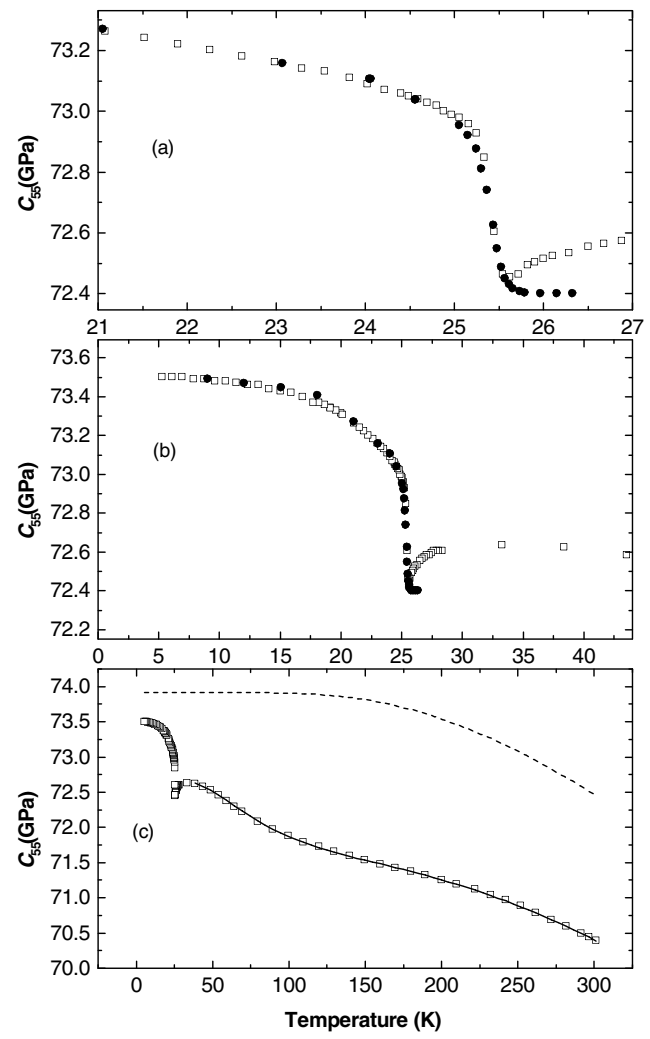

FIG. 3. The elastic constant $C_{55}$ of $\mathrm{U}_{2} \mathrm{Rh}_{3} \mathrm{Si}_{5}$ vs temperature for different temperature scales. (a),(b) The open symbols represent the experimental elastic constant and the solid symbols represent the intensity of a neutron scattering Bragg reflection, such intensity being proportional to the square of the ordered magnetic moment. (c) The symbols represent the elastic constant data, the solid line is a fit using Eq. (3), and the dashed line represents the background elastic constant, Eq. (4). 
The latter measurements present the square root of the intensity of a Bragg reflection, normalized to its value at $9 \mathrm{~K}$, which directly reflects the temperature dependence of the ordered moment in the antiferromagnetic phase. We assume that below the ordering transition each elastic constant can be described by

$$
\frac{C_{i j}(T)-C_{i j}^{o}}{C_{i j}(9 \mathrm{~K})-C_{i j}^{o}}=\frac{I(T)}{I(9 \mathrm{~K})} .
$$

Here, $I(T) / I(9 \mathrm{~K})$ is the normalized (301) Bragg reflection intensity from the neutron diffraction results of Ref. [10]. This assumption says that the change in the elastic constant, relative to some background elastic constant $C_{i j}^{o}$, is proportional to the square of the ordered moment on the $\mathrm{U}$ atoms. The only adjustable parameter for this " $\mathrm{fit}$ " is $C_{i j}^{o}$ for each elastic constant. Figs. 3(a) and 3(b) give $C_{55}(T)$ computed from Eq. (1). As can be seen, the correspondence is excellent. Good agreement for all the diagonal elastic constants was found using such a scheme, with just a single $C_{i j}^{o}$ for each modulus. This agreement shows that strong spin-lattice coupling dominates the elastic constant behavior below the transition temperature.

The third point requires a longer discussion. None of the moduli of Fig. 2 show strong softening well above the transition as might be expected, for example, for a cooperative Jahn-Teller transition [20]. However, all of the elastic constants show some unusual behavior considerably above the phase transition. For $C_{55}$, as shown in Fig. 3(c), this behavior involves a bend upward at about $100 \mathrm{~K}$. Such concave temperature dependence is unusual. We attribute the unconventional behavior well above the transition to a coupling of the elastic waves to CEF levels [21]. The quasiorthorhombic symmetry is expected to completely lift the angular-momentum degeneracy of the $\mathrm{U} 5 f^{2}\left({ }^{3} \mathrm{H}_{4}\right)$ ion [22]. Unfortunately, little is known about the CEF levels for the present case and thus it seems useless to try to fit the present results to orthorhombic symmetry. As an approximate description we treat the case for cubic symmetry. The local cluster of $\mathrm{Si}$ and $\mathrm{Rh}$ atoms surrounding the $\mathrm{U}$ ion provide some support for this approximation as does the near elastic isotropy mentioned earlier. We start with the Hamiltonian

$$
H=B_{4}\left(O_{4}^{0}+5 O_{4}^{4}\right)+\eta_{3} e_{x y}\left(J_{x} J_{y}+J_{y} J_{x}\right) .
$$

Here, the first term describes the electronic states of the ${ }^{3} \mathrm{H}_{4}$ ion in the crystalline electric field and the second term treats the coupling of these states to the strain $e_{x y}$ corresponding to the elastic constant $C_{55}$. The parameter $B_{4}$ is a crystal-field parameter and the $O$ s are Stevens operators $[23,24]$. We neglect higher order terms in the crystal field for the present approximate description. Diagonalization of the first term only partially lifts the ninefold degeneracy of the ${ }^{3} \mathrm{H}_{4}$ ion, resulting in a singlet, a doublet, and two triplets. The form of the second term is determined by symmetry [21] and gives the coupling of the crystal-field levels to the strain $e_{x y} ; \eta_{3}$ is the strain-ion coupling constant. The $J$ 's are angular-momentum operators. The Hamiltonian, $H$, was diagonalized to find the energy levels $E_{i}$ in terms of $B_{4}$, and their strain dependence in terms of $\eta_{3}$. The elastic constant, $C_{55}$, is given by

$$
C_{55}(T)=C_{\mathrm{bg}}(T)+\Delta C_{55}(T) .
$$

Here, we use the well-known Varshni expresson [25] for the background (bg) elastic constants

$$
C_{\mathrm{bg}}(T)=C_{0}-\frac{s}{\exp \left(T_{E} / T\right)-1} .
$$

The effect of coupling to the CEF levels is obtained from $\Delta C_{55}=\partial^{2} F / \partial e_{x y}^{2} \quad$ with $\quad F=-N k_{B} T \ln (Z)$, where $N\left(1.227 \times 10^{28} / \mathrm{m}^{3}\right)$ is the number of $\mathrm{U}$ atoms per volume, and $Z=\sum_{i=1}^{9} \exp \left(-E_{i} / k_{B} T\right)$ is the partition function. The result is

$$
\Delta C_{55}=N\left[\frac{1}{Z}\left(\sum_{i} \frac{\partial^{2} E_{i}}{\partial e_{x y}^{2}} \exp \left(-E_{i} / k_{B} T\right)-\frac{1}{k_{B} T} \sum_{i}\left(\frac{\partial E_{i}}{\partial e_{x y}}\right)^{2} \exp \left(-E_{i} / k_{B} T\right)\right)+\left(\frac{\Sigma_{i} \frac{\partial E_{i}}{\partial e_{x y}} \exp \left(-\frac{E_{i}}{k_{B} T}\right)}{Z}\right)^{2}\right] .
$$

The nine energy levels $E_{i}$ and their strain derivatives are obtained from the diagonalization of Eq. (2). $\Delta C_{55}$ depends only on the two parameters $B_{4}$ and $\eta_{3}$, which are determined by fitting the data. The last term in Eq. (5) gives zero contribution. Figure 3(c) gives the fitting results. The dashed line represents Eq. (4), showing the normal background temperature dependence. The solid line through the data points results from fitting Eq. (3) to the data above the phase transition. The fit is excellent and the fitting parameters for $\Delta C_{55}$ are: $B_{4}=-20.8 \times 10^{-3} \mathrm{meV}$ and $\eta_{3}=$ $21 \mathrm{meV}$. (The parameters of the bg elastic constant are: $C_{o}=73.9 \mathrm{GPa}, s=18.3 \mathrm{GPa}$, and $T_{E}=783 \mathrm{~K}$, representing typical behavior.) The temperature dependences of the other elastic constants well above the transition are also accounted for by this procedure with the same value of $B_{4}$, but of course different values of $\eta_{3}$. In some cases it is necessary to take into account coupling to the shear strain corresponding to $C_{11}-C_{12}$ [21].

It is of interest to compare the results to $\mathrm{PrSb}$ where the $\mathrm{Pr}^{3+}$ ion has the same electronic configuration $\left({ }^{3} \mathrm{H}_{4}\right)$. For PrSb, neutron scattering [26] gives $B_{4}=-6.5 \times$ $10^{-3} \mathrm{meV}$, not very different from the present results, with $B_{4}$ for the $\mathrm{U}$ ion being greater in magnitude as expected on going from $4 f$ to $5 f$ compounds. Our value of $\eta_{3}$ is of the same order of magnitude [21] as that found for $\mathrm{PrSb}$, but is about a factor of 5 too small to produce a structural phase transition (i.e., drive $C_{55}$ to zero) [27]. Given the dearth of information about CEF levels in $\mathrm{U}_{2} \mathrm{Rh}_{3} \mathrm{Si}_{5}$ it is worth noting that $B_{4}=-20.8 \times 10^{-3} \mathrm{meV}$ gives the following CEF levels: singlet at $0 \mathrm{~K}\left(\Gamma_{1}\right)$, triplet at $203 \mathrm{~K}$ $\left(\Gamma_{4}\right)$, doublet at $347 \mathrm{~K}\left(\Gamma_{3}\right)$, and a triplet at $781 \mathrm{~K}\left(\Gamma_{5}\right)$. A positive value of $B_{4}$, which would give $\Gamma_{5}$ as the lowest 
level, gives qualitatively different behavior for $C_{55}$ and does not correspond to the data. There are no published reports of CEF levels in $\mathrm{U}_{2} \mathrm{Rh}_{3} \mathrm{Si}_{5}$ with which to compare the present results, although unpublished neutron scattering results indicated a broad level in the $20-30 \mathrm{meV}$ range [11] that may be due to CEF levels, and would be consistent with the present results. The ultrasonically-derived levels, based only on the first term for a cubic crystal field, are expected to be split by the lower site symmetry of the present case.

Based upon our evaluation of the elastic constant measurements, we attribute the dramatic first-order nature of the magnetic transition to a strong spin-lattice coupling involving the CEF levels. In our metallic $\mathrm{U}_{2} \mathrm{Rh}_{3} \mathrm{Si}_{5}$ case a $\Gamma_{1}$ (nonmagnetic singlet) ground state becomes entwined with a nearby magnetic triplet $\left(\Gamma_{4}\right.$ or $\left.\Gamma_{5}\right)$ due to the itinerant exchange field, which removes its degeneracy and forces a magnetic level in close proximity to $\Gamma_{1}$. As this level becomes increasingly populated $(T \rightarrow 25 \mathrm{~K})$, the spin-lattice coupling enhances the downward splitting, and a "catastrophic" or "bootstrap" transition results [28-30]. Note that the phase transition is not driven by quadrupole ordering, but is magnetically driven with a large unit cell volume expansion as $T$ decreases $[8,9]$ due to the strong spin-lattice coupling.

In summary, the complete nine-component elastic tensor has been determined through the first-order phase transition in quasiorthorhombic $\mathrm{U}_{2} \mathrm{Rh}_{3} \mathrm{Si}_{5}$. This unusual achievement may be due in part to the absence of a change in crystal symmetry at the transition [10]. Based on the high-quality fit of the $C_{i j}$ to the observed resonance frequencies - and the high quality of the resonances - in the antiferromagnetic phase, we conclude that domains (magnetic or structural) play little part in the material's elastic response. All elastic constants show abrupt changes at the transition at $25.5 \mathrm{~K}$. Below the transition all diagonal moduli are strongly correlated with the square of the ordered magnetic moment, demonstrating strong spinlattice coupling. The transition is not preceded at higher temperatures with strong softening of any of the elastic constants over an extended temperature range as would be the case for a soft-mode transition. Anomalies in the temperature dependence of the elastic constants above the phase transition are explained in terms of coupling to CEF levels, but this coupling is too weak to produce a structural phase transition. The results suggest that the first-order phase change is magnetically driven by a bootstrap mechanism involving the ground state singlet and a magnetically active CEF level.

[1] P. Thalmeier and G. Zwicknagl, in Handbook of the Physics and Chemistry of Rare Earths, edited by K. A. Gschneidner, J.-C.G. Bunzil, and V.K. Pecharsky (Elsevier, Amsterdam, 2005), Vol. 34, Chap. 219.
[2] J. Flouquet, in Progress in Low Temperature Physics, edited by D. Brewer (North Holland, Amsterdam, to be published).

[3] See Ref. [1], p. 13.

[4] K. Andres, D. Davidov, P. Dernier, F. Hsu, W. A. Reed, and G. J. Nieuwenhuys, Solid State Commun. 28, 405 (1978); H. R. Ott, K. Andres, and P. H. Schmidt, Physica B\&C 102, 148 (1980).

[5] N. Lingg, D. Maurer, V. Müller, and K. A. McEwen, Phys. Rev. B 60, R8430 (1999).

[6] D. F. McMorrow, K. A. McEwen, U. Steigenberger, H. M. Rønnow, and F. Yakhou, Phys. Rev. Lett. 87, 057201 (2001).

[7] E. Hickey, B. Chevalier, P. Gravereau, and J. Etourneau, J. Magn. Magn. Mater. 90-91, 501 (1990).

[8] B. Becker, S. Ramakrishnan, A. A. Menovsky, G. J. Nieuwenhuys, and J. A. Mydosh, Phys. Rev. Lett. 78, 1347 (1997).

[9] T. Takeuchi, T. Yamada, Y. Miyako, K. Oda, K. Kindo, B. Becker, S. Ramakrishnan, A. A. Menovsky, G. J. Nieuwenhuys, and J.A. Mydosh, Phys. Rev. B 56, 10778 (1997).

[10] R. Feyerherm, C. R. Wiebe, B. D. Gaulin, M. F. Collins, B. Becker, R. W. A. Hendrikx, T. J. Gortenmulder, G. J. Nieuwenhuys, and J. A. Mydosh, Phys. Rev. B 56, 13693 (1997).

[11] F. Galli, Ph.D. thesis, Chap. 7, Leiden University, 2002 (unpublished).

[12] B. Becker, Ph.D. thesis, Chap. 4, Leiden University, 1997 (unpublished).

[13] A. Migliori, J. L. Sarrao, W. M. Visscher, T. M. Bell, Ming Lei, Z. Fisk, and R. G. Leisure, Physica B (Amsterdam) 183, 1 (1993).

[14] B. Lüthi and W. Rehwald, in Structural Phase Transitions I (Springer, Berlin, 1981), pp. 131-184.

[15] B. Lüthi, Physical Acoustics in the Solid State (Springer, Berlin, 2005).

[16] H. Ledbetter, in Handbook of Elastic Properties of Solids, Liquids, and Gases (Academic, San Diego, 2001), p. 57.

[17] H. Ledbetter, S. Chevacharoenkul, and R. Davis, J. Appl. Phys. 60, 1614 (1986).

[18] L. D. Landau and E. Liftshitz, Statistical Physics (Pergamon, Oxford, 1980), Chap. XIV, p. 451.

[19] W. Rehwald, Adv. Phys. 22, 721 (1973).

[20] R. L. Melcher and B. A. Scott, Phys. Rev. Lett. 28, 607 (1972).

[21] B. Lüthi, M. E. Mullen, and E. Bucher, Phys. Rev. Lett. 31, 95 (1973).

[22] U. Walter, J. Phys. Chem. Solids 45, 401 (1984).

[23] M. T. Hutchings, in Solid State Physics, edited by F. Seitz and D. Turnbull (Academic, New York, 1964), Vol. 16.

[24] U. Walter, Z. Phys. B 62, 299 (1986).

[25] Y. P. Varshni, Phys. Rev. B 2, 3952 (1970).

[26] K. C. Turberfield, L. Passell, R. J. Birgenau, and E. Bucher, J. Appl. Phys. 42, 1746 (1971).

[27] M.E. Mullen, B. Lüthi, P. S. Wang, E. Bucher, L. D. Longinotti, J. P. Maita, and H. R. Ott, Phys. Rev. B 10, 186 (1974).

[28] M. Blume, Phys. Rev. 141, 517 (1966).

[29] S. J. Allen, Phys. Rev. 167, 492 (1968).

[30] P. Erdös and J. M. Robinson, The Physics of Actinide Compounds (Plenum, New York, 1983), Chap. 3. 